\title{
The Possibilities of Nonlinear Ultrasonic Spectroscopy for the NDT in Civil Engineering
}

\author{
M. Manychova \\ Department of Building Structures, Faculty of Civil Engineering, Brno University of Technology, Brno, Czech \\ Republic. \\ * Coresponding author: manychova.m@fce.vutbr.cz
}

\begin{abstract}
Current methods of non-destructive ultrasonic material testing are based on the analysis of elastic wave reflection, absorption and interference. These methods are difficult to apply to inhomogeneous building materials showing tiny cracks and defects distributed throughout the specimen bulk, or in the cases where the defect size is comparable with the wavelength. Analysis of these phenomena occurring in intricate shapes is also difficult. To cope with such problems, application of wave propagation related nonlinear effects and higher harmonic signal generation in the defect vicinity is advisable. Due to the presence of defects, the atomic potential energy ceases to be exactly harmonic. Second and third harmonic frequencies arise. In this domain, methods employing the nonlinear acoustic spectroscopy apply. These novel defectoscopic methods are based on the nonlinear behaviour of current defects and inhomogeneities regarding the elastic wave propagation processes. Unlike the electromagnetic and acoustic emission methods, which only allow the localization of currently emerging cracks and defects, the non-linear ultrasonic defectoscopy is all-defect-sensitive, thus constituting a method applicable to characterizing the quality and reliability of materials.
\end{abstract}

KEY WORDS: Nonlinear ultrasonic spectroscopic methods, inhomogeneous materials, material degradation, ceramic specimens, structural integrity

\section{INTRODUCTION}

Due to their assumedly higher sensitivity and more accurate quality and reliability characterization capacity, the non-linear ultrasonic spectroscopy methods are ranked among the most promising material quality and reliability characterization tools. Detailed studies of dynamic non-linearities and hysteresis in inhomogeneous media have shown that the occurrence of mesoscopic elements in the material structure gives rise to strongly non-linear dynamic phenomena accompanying the elastic wave propagation (Van Den Abeele at al. 2000, Johnson, 1999). These non-linear effects are observed in the course of the degradation process much sooner than any degradation-induced variations of linear parameters (propagation velocity, attenuation, elastic moduli, rigidity, etc.). Non-linear parameters have proved to be very sensitive to the presence of any inhomogeneities and progressing degradation of the material structure. Non-linear wave methods thus open new horizons to the acoustic non-destructive testing: they provide higher sensitivities, application speed and easy interpretation. 
One of the fields in which a wide application range of non-linear acoustic spectroscopy methods may be expected is civil engineering. Poor material homogeneity and, in some cases, the shape complexity of some units used in the building industry, are heavily restricting the applicability of "classical" ultrasonic methods (Macecek, 2004). Some of the non-linear acoustic defectoscopy methods are less susceptible to the aforementioned restrictions and one may expect them to contribute to a great deal to further improving the defectoscopy and material testing methods in civil engineering.

\section{NONLINEAR ULTRASONIC SPECTROSCOPY}

Non-resonance methods are used to study suppressed resonance specimens. These methods can be split into two groups:

- measurements using a single harmonic ultrasonic signal (a single frequency $\mathrm{f}_{1}$ )

- measurements using multiple harmonic ultrasonic signals (usually, two frequencies $\mathrm{f}_{1}, \mathrm{f}_{1 .}$ )

These methods analyse the effect of non-linearities on the acoustic signals propagating through them.

\subsection{Single Harmonic Ultrasonic Signal Measurement Method}

In this case, where a single exciting frequency $f_{1}$ is used (Fig. 1), the non-linearity gives rise to other harmonic signals, whose frequencies $f_{v}$ obey the Fourier series formulas:

$$
f_{v}=n f_{1} \quad \mid n=0,1,2, \ldots \infty
$$
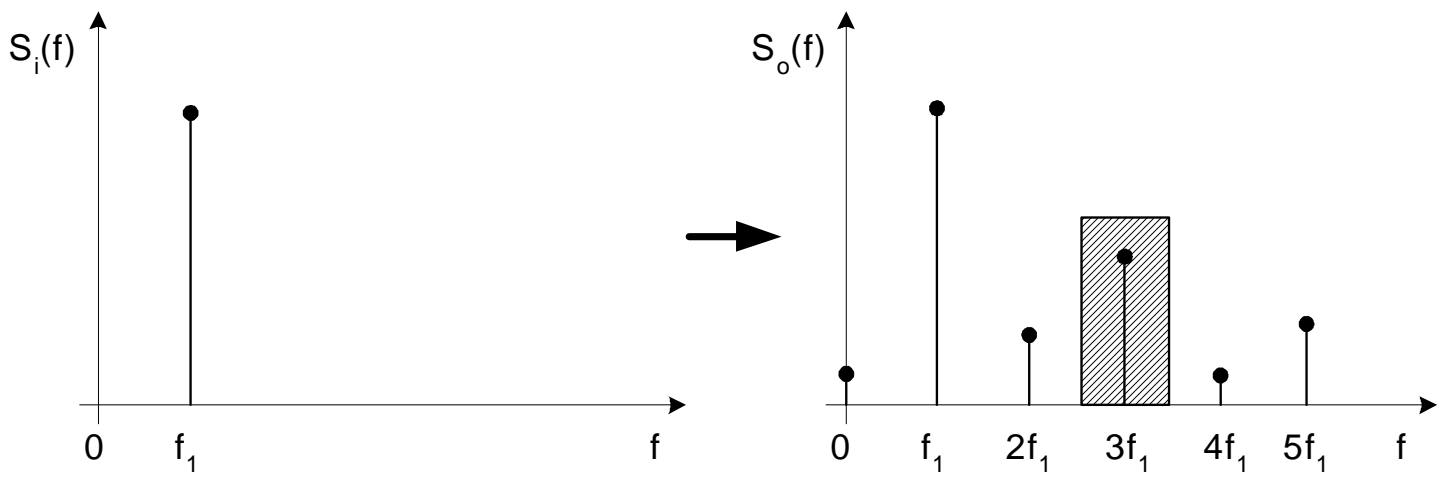

Figure 1: Frequency spectrum of a non-linear medium response.

In general, these frequency component amplitudes are falling when the harmonic order natural number, $n$, is increasing. If the non-linearity effect is not entirely symmetrical, a lowamplitude second or higher even-numbered harmonic components can arise, 
whose amplitudes may be much lower than those of the odd-numbered ones. Among these emerging components, the third harmonic is the most distinctive one. Therefore, its amplitude is the one which is most frequently evaluated (Hajek \& Sikula., 2005).

\section{EXPERIMENT}

Based on the results of our studies of building material non-linear behaviour, a measuring apparatus for diagnosing the structure integrity has been assembled.

\subsection{Experimental Arrangement}

A single harmonic ultrasonic signal method was applied. The measuring apparatus consisted of two principal parts, namely, a transmitting unit and a receiving unit.

The transmitting units consist of three functional blocks: a controlled-output-level harmonic signal generator, a low-distortion $100 \mathrm{~W}$ power amplifier and a low-pass output filter designed to suppress higher harmonic components and ensure high purity of the exciting harmonic signal.

The main chain of the receiving unit includes an input amplifier with filters designed to minimize the receiving chain distortion and a band-pass filter amplifier. Having been amplified, the sensor output signal is sampled in a DL920 transient recorder, to be subsequently saved in a computer memory for evaluation, see Fig. 2.

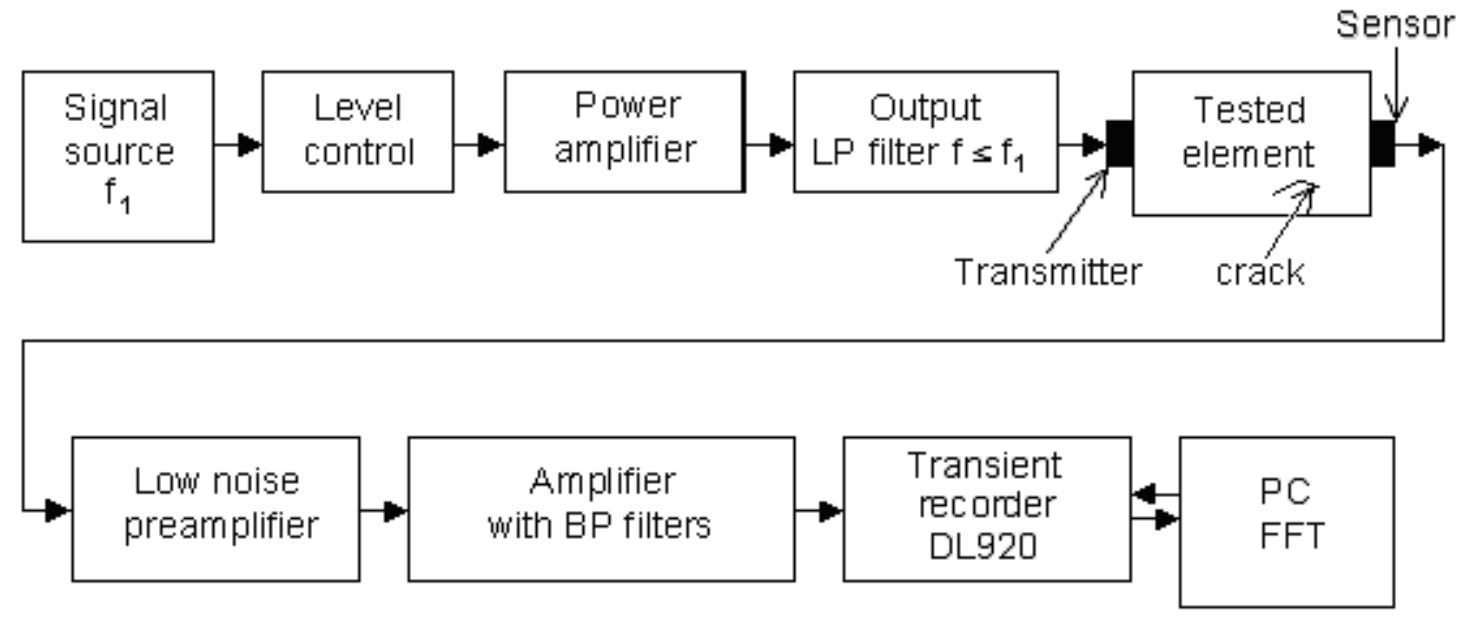

Figure 2: Block scheme of the measure equipment.

To interpret the recorded data properly, each of the measuring instruments must answer the demands of high linearity. For the purpose of improving the reliability and accuracy of the nonlinear experiments and minimizing the error effects the attention was focused to transmission between transmitter and sensors. A program package to control the measuring process, the data processing, and evaluation makes for an indispensable tool. The measurement results were represented in the form of frequency spectra.

The single-harmonic-signal non-linear ultrasonic spectroscopy method was applied to the tests of cladding elements which had been made of secondary materials. The specimens are prototypes of BIII group, fly-ash-argilic-body based ceramic cladding elements prepared 
to ČSN EN 14411. Two specimen types were studied, namely, A and B. The B-type was represented by two specimens: B1 specimen, featuring an intact structure, and B2 specimen which contained a visible crack, see Fig. 3.

Two mutually perpendicular orientations of the exciter and the sensor (position 1 and position 2, see Fig. 3) were measured. An ultrasonic generator with a frequency $f_{1}=29 \mathrm{kHz}$ was used as a transmitter (exciter), to generate a harmonic ultrasonic wave in the specimen (Korenska \& Manychova, 2008) Intact specimens were used to check whether or not the fly-ash-argilic body containing structure created the sources of signal propagation related to the non-linear phenomena. The effect of the structure defect (crack) on the propagating signal properties was studied on the B2 specimen.

The influence of exciter voltage values on transmission characteristic changes was investigated and their frequency spectra were analyzed.

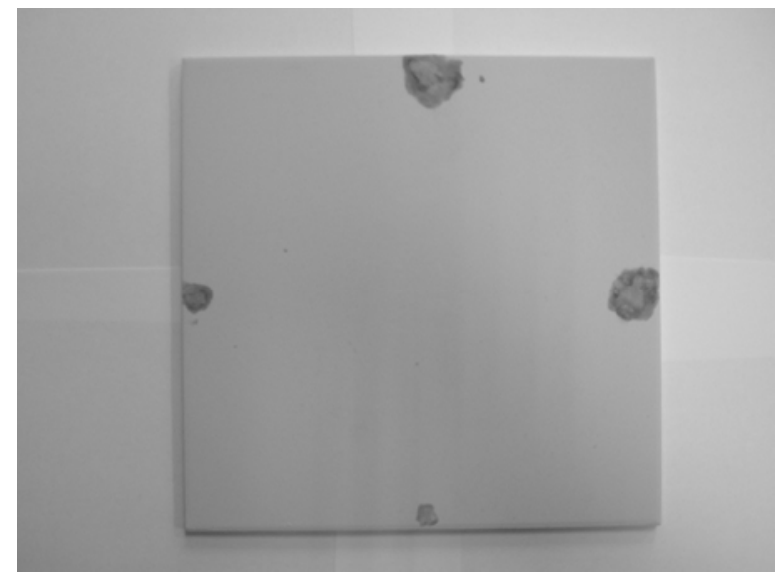

Specimen A

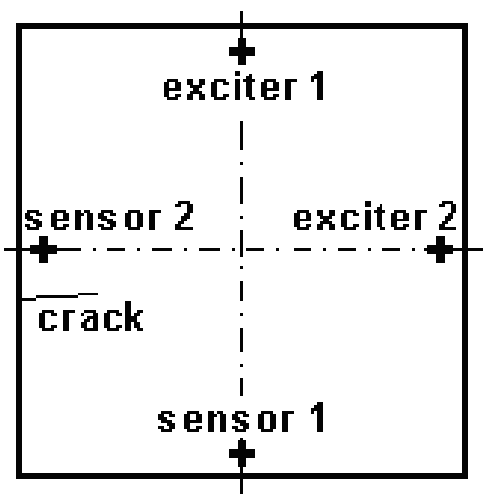

Specimen B2

Figure 3: Cladding element specimens under test.

\subsection{Measurement Results}

The following figures represent frequency spectra of the exciting frequency response for three different exciting voltage values. The graph in Fig. 4 represents the measurement results (position 1) of the intact sample, denoted A. From the graph a progressive decrease 
of amplitudes is apparent for all voltage values. An analogous result, apart from nonlinear effects, was attained in position 2 as well.

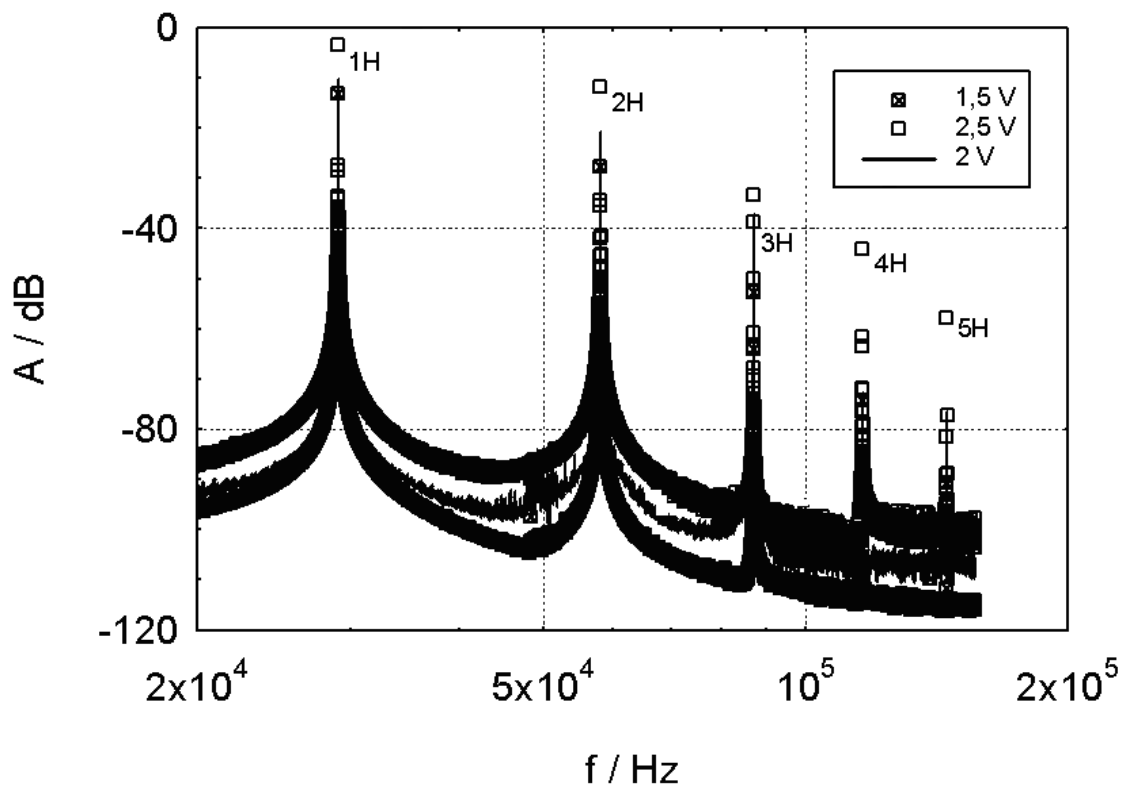

Figure 4: Frequency spectra of intact specimen A, orientation 1.

The next graph in Fig. 5 represents the measurement result of the intact sample, denoted B1. In this case analogous results, without the nonlinear effect, were reached for both positions of exciter and sensor.

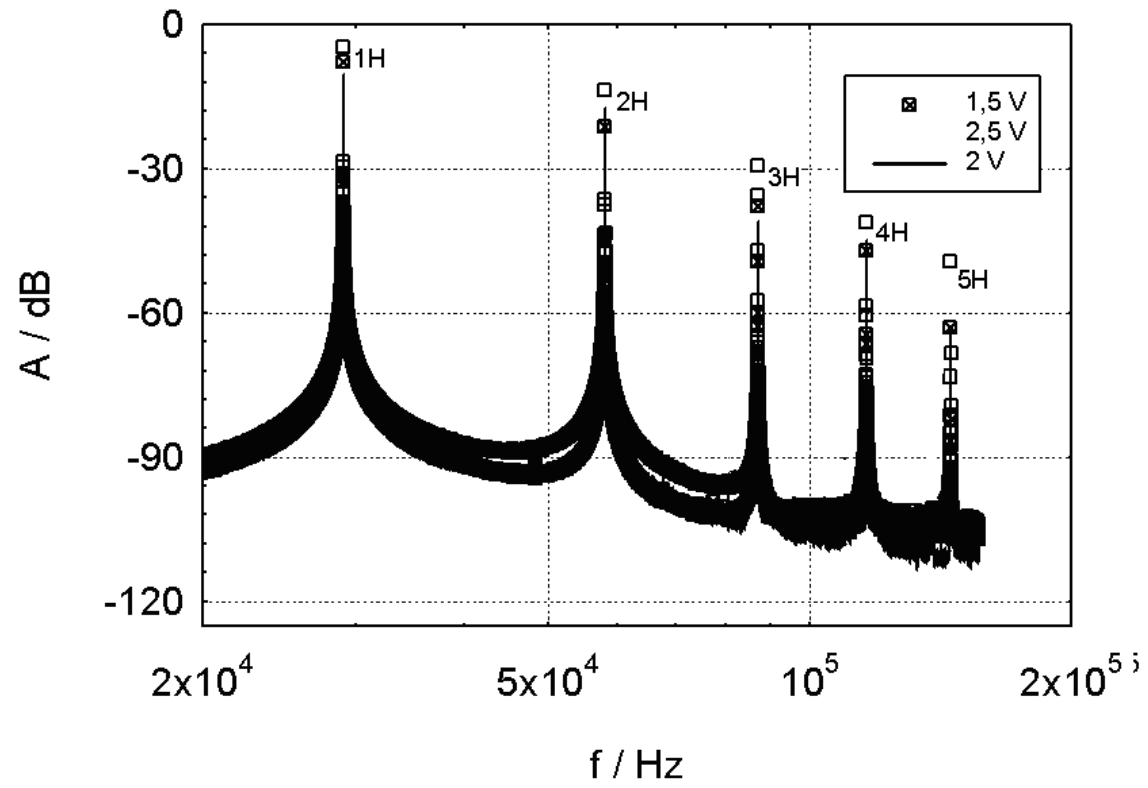

Figure 5: Frequency spectra of intact specimen B1, orientation 1. 
Figs. 6 and 7 correspond to the B2 specimen with a visible crack. The graph in Fig. 5 represents the position 1 measurement result. The third harmonic component dominates in the frequency spectrum and the amplitude of the fifth harmonic is higher in comparison with the fourth harmonic component for all exciter voltage values.

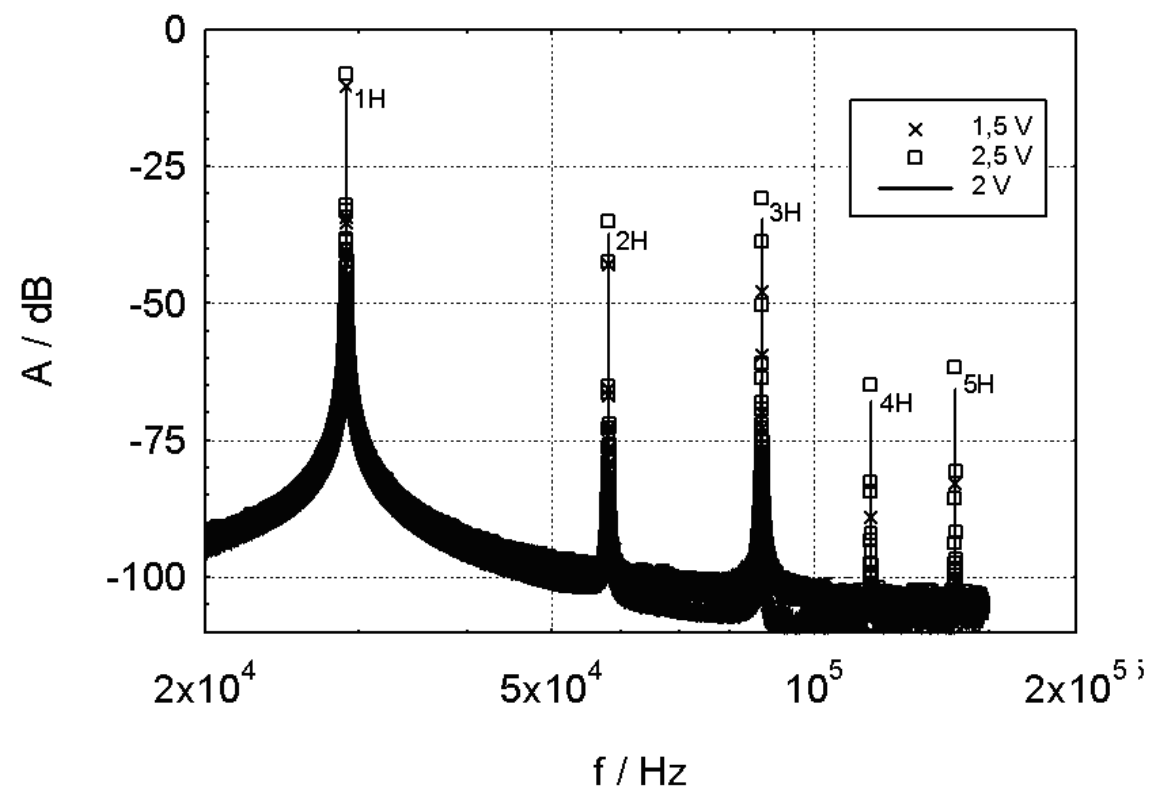

Figure 6: Frequency spectra of damage specimen B2, orientation 1.

The last graph, Fig. 7, shows the measurement result of the same specimen B2 for orientation 2; sensor was placed near the crack. In this case the second harmonic component dominates for all voltage values. The amplitudes of the fourth and fifth reach almost the same values.

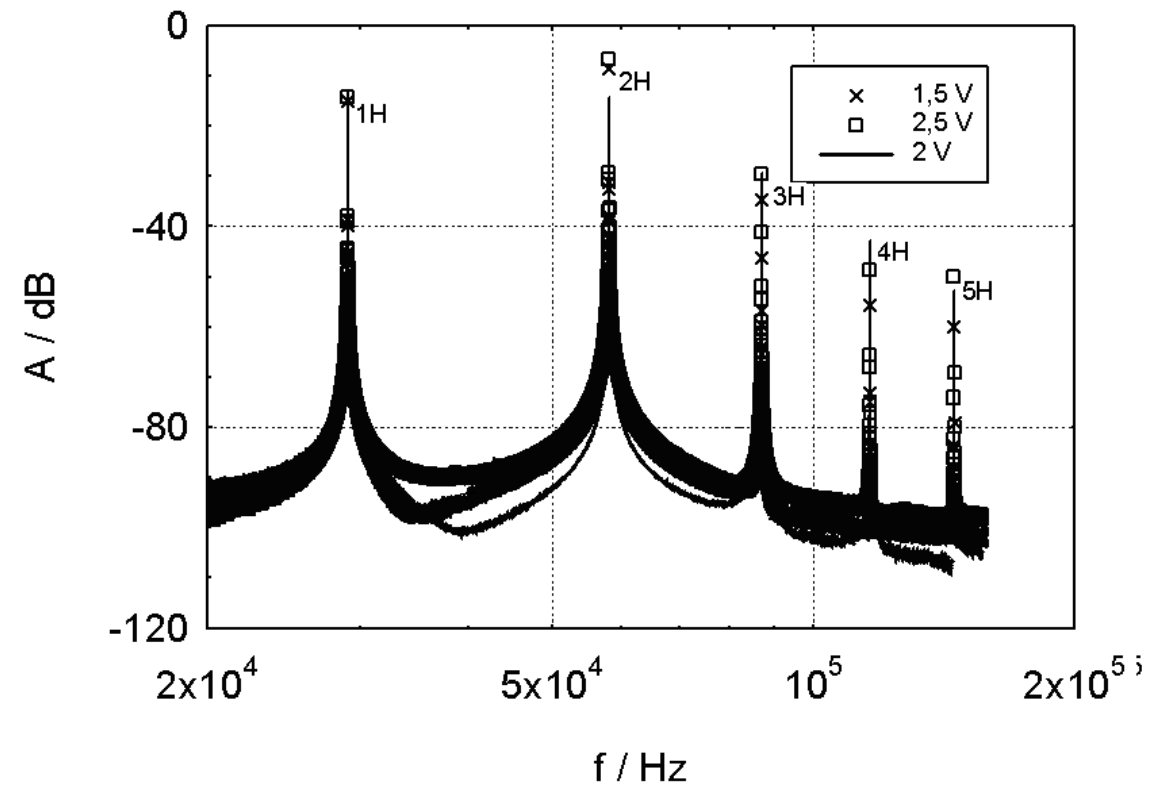

Figure 7: Frequency spectra of damage specimen B2, orientation 2. 


\section{CONCLUSION}

The single-harmonic ultrasonic signal method was applied to advanced building materials made with the use of secondary materials. Prototypes of BIII group, fly-ash-argilic-body based ceramic cladding elements prepared to ČSN EN 14411, were tested.

Non-linear effects were analyzed for both an intact specimen and a specimen containing a defect (a crack).

a) Intact specimens were used to check whether or not the specimen inhomogeneous structure creates the source of signal propagation related non-linear phenomena. Our measurements have furnished evidence that the effect of a material's inhomogeneity is very low in the case of non-linear ultrasonic spectroscopy, its non-linear effect being substantially lower than that of common defects. The transfer function of these specimens does not feature any non-linear phenomena. The higher harmonic component amplitudes fell down progressively with an increase in the serial number $n$. Two mutually perpendicular exciter-to-sensor orientation based measurements gave identical results.

b) In the apparent crack specimen case, we focused on the transfer function variations. The transfer functions as obtained from both exciter-to-sensor configurations showed a nonlinearity, which was due to the specimen structure defect. The transfer function curves differed from one another depending on the sensor-to-crack position. If the sensor was farther away from the crack, the odd-numbered (3rd and 5th) harmonic frequency amplitudes were below those of the foregoing even-numbered (2nd, 4th) harmonic components, respectively. If the sensor was fitted near the crack, an anomaly was observed, namely, the second harmonic amplitude exceeded that of the first (exciting) frequency amplitude. The fourth and fifth harmonic amplitudes reached comparable values.

\section{ACKNOWLEDGEMENTS}

This research was supported by the research project MSM 0021630511.

\section{REFERENCES}

Van Den Abeele, K. E.-A., P. A. Johnson, and A. Sutin, 2000. Nonlinear Elastic Wave Spectroscopy (NEWS) techniques to discern material damage. Part I: Nonlinear Wave Modulation Spectroscopy (NWMS), Research on NonDestructive Evaluation 12, pp. 17-30.

Van Den Abeele, Jan Carmeliet, James A. Ten Cate, Paul A. Johnson, 2000. Nonlinear Elastic Wave Spectroscopy (NEWS) Techniques to Discern Material Damage. Part II: Single Mode Nonlinear Resonance Acoustic Spectroscopy. Research on NonDestructive Evaluation 12, pp. 31- 42.

Hajek, K., Sikula, J., 2005. Testing of Low-Current Contacts Quality and Reliability by Using Third Harmonic Distortion. IEEE Trans. on Components and Packaging Technologies 28, pp.717 - 720 .

Johnson, P. A., 1999. The new wave in acoustic testing. The J. Inst. Materials 7, pp. 544-546.

Korenska, M, Manychova, M., 2008. Nonlinear Ultrasonic Spectroscopy Used to Detection of Ceramic Structure Damage. Nonlinear Acoustics- Fundamentals and Applications, 
ISNA18, pp. 541-544, Ed. Bengt O. Enflo, Claes M. Hedberg, Leif Kari, American Institute of Physics, New York 2008.

Korenska, M, Pazdera, L., Ritickova, L., 2001. Resonant inspection - Interesting nondestructive testing tools for determine quality of tested specimen. $6^{\text {th }}$ International Conference of the Slovenian-Society-for-Non-Destructive-Testing, pp. 45-48, Ed. Grum j., Lovšin N., Slovenian Society for NDT.

Korenska, M., Chobola, Z., Sokolar, R., Mikulkova, P., Martinek, J., 2006. Frequency Inspection as an Assessment Tool for the Frost Resistance of Fired Roof Tiles. CeramicsSilikáty, vol. 50, iss. 3, pp. 185 - 192.(2006).

Macecek, M., 2003. Ultrasonic Concrete Testing. $33^{\text {rd }}$ International Conference Defektoskopie 2003, pp. 117-132, Ed. Mazal P. 